\title{
On the entanglement entropy of spacetime and matter based on the action in general relativity
}

\author{
Shujuan $\mathrm{Liu}^{1}$ and Hongwei Xiong ${ }^{1,2, *}$ \\ ${ }^{1}$ College of Science, Zhejiang University of Technology, Hangzhou 31023, China \\ ${ }^{2}$ Wilczek Quantum Center, Department of Physics and Astronomy, \\ Shanghai Jiao Tong University, Shanghai 200240, China
}

(Dated: February 13, 2019)

\begin{abstract}
We consider the conjecture of the relationship between the entanglement entropy of spacetime, matter and the action in Einstein's general relativity. Our analysis suggests the possibility of regarding the entanglement entropy of spacetime and matter as the dimensionless action in general relativity by using Planck units. In this case, the action principle originates from the maximization of the entanglement entropy. We also show that the fundamental property of entanglement entropy leads to attractive characteristic of gravitational force for classical particles.
\end{abstract}

*Electronic address: xionghw@zjut.edu.cn 


\section{INTRODUCTION}

The concept of quantum entanglement is playing ground-breaking role in the understanding of spacetime, gravity, black hole and the whole universe [1-12]. It is well known that the entanglement entropy emerges naturally from the consideration of a subsystem for the whole quantum entangled system. In the present work, we consider the relationship between the entanglement entropy of spacetime, matter and the action in Einstein's general relativity.

We consider a sphere of radius $r$. The surface $\Sigma_{S}$ of this sphere divides the whole universe into two systems $A$ and $B$ shown in Fig. 1, i.e., the interior of the sphere and the external environment. Assuming the quantum state of the whole universe including spacetime is $\left|\Psi_{A B}\right\rangle$, the entanglement entropy for system $A$ is [13]

$$
S_{A}=-\operatorname{Tr}\left[\rho_{A} \log \rho_{A}\right]
$$

Here $\rho_{A}=\operatorname{Tr}_{B}\left(\rho_{A B}\right)$ is the reduced density matrix with $\rho_{A B}=\left|\Psi_{A B}\right\rangle\left\langle\Psi_{A B}\right|$ the density matrix for the pure state $\left|\Psi_{A B}\right\rangle$. For flat spacetime and the situation of $r>>l_{p}$ with $l_{p}$ the Planck length, it is possible that this entanglement entropy $S_{A}$ is of the order of $4 \pi r^{2} / l_{p}^{2}$ by regarding $l_{p} \times l_{p}$ as the smallest spacetime cell on the spherical surface [14-16]. If there is matter distribution inside the sphere shown in Fig. 1, it is expected that there would be extra entanglement entropy due to matter distribution and the spacetime curvature because of the existence of matter. The purpose of this work is to try to find a way to calculate these extra entanglement entropies. It is shown the possibility of close connection of this entanglement entropy with the action of general relativity. We show further that the action principle of general relativity can be interpreted as the maximization of entanglement entropy. This also provides a way to interpret the attractive characteristic of gravitational force.

The paper is organized as follows. In Sec. II, we consider the entanglement entropy for a particle inside the sphere. In Sec. III, we consider the entanglement entropy for spacetime. In Sec. IV, we consider the connection between entanglement entropy and action principle in general relativity. We also consider the attractive characteristic of gravitational force for classical particles, based on the fundamental properties of entanglement entropy. In the last section, we give a brief summary and discussion of our work. 


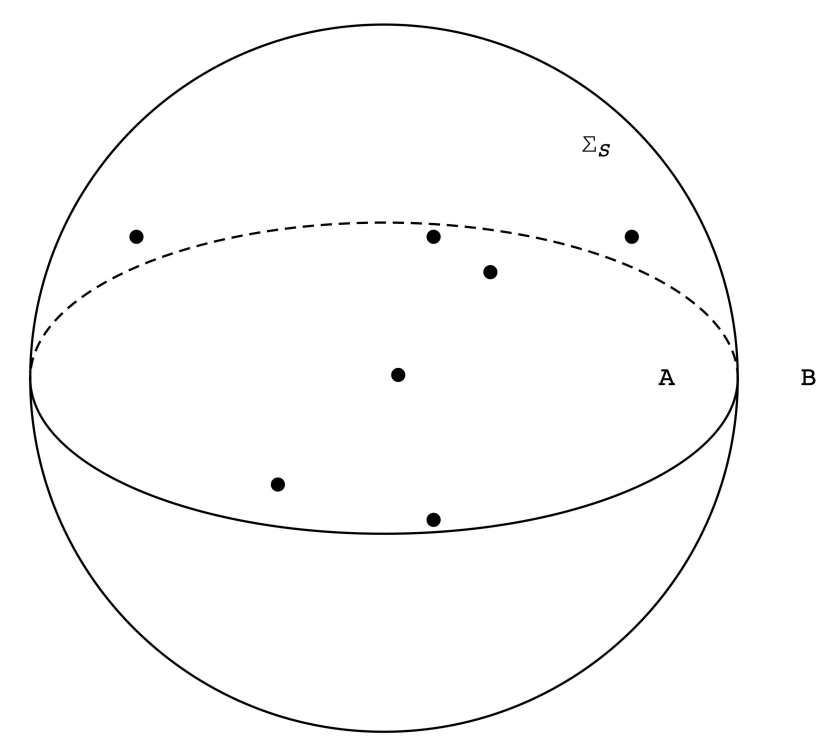

Figure 1: Schematic diagram of two systems $A$ and $B . \Sigma_{S}$ denotes the spherical surface.

\section{ENTANGLEMENT ENTROPY FOR DISPLACEMENT OF A PARTICLE AND THE GENERALIZATION TO CURVED SPACETIME}

We consider the situation in Fig. 2 that there is a particle of mass $M$ in the interior of a sphere of radius $r$. This particle moves along a path from $A$ to $B$. Assume that the overall spatial length of this path is $l_{A B}$. Based on the work in Ref. [17], there would be an increase of entropy given by

$$
S_{M} \sim l_{A B}
$$

This entropy for displacement is used in Ref. [18] to understand the direction of gravitational force. At first sight, we may think that this is nonsense because there is no friction for this particle due to spacetime. However, if we regard $S_{M}$ as an entanglement entropy, we may understand this formula.

We consider the quantum state $|\Psi\rangle$ including both spacetime and $M$. As shown in Fig. 2, the spherical surface $\Sigma_{S}$ separates the system into two systems $C$ and $D$. The entanglement entropy of $C$ can be written as

$$
S_{\text {entangle }}=S_{0}+S
$$

Here $S_{0} \sim 4 \pi r^{2} / l_{p}^{2}$ is the entanglement entropy without matter in the sphere for flat spacetime. $S$ is the extra entanglement entropy due to the presence of the particle $M$. In this case, we may understand Eq. (2), although we do not know the coefficient yet and this is 


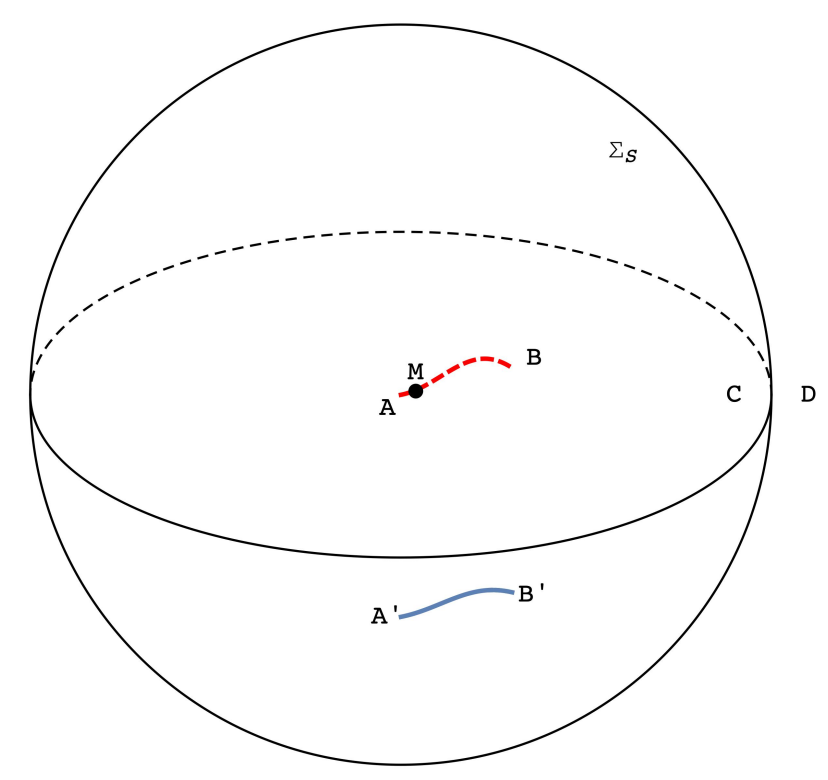

Figure 2: Schematic diagram of the path of a particle $M$. The dashed line inside the sphere shows the path of $\mathrm{M}$ from $\mathrm{A}$ to $\mathrm{B}$, while the solid line on the sphere from $A^{\prime}$ to $B^{\prime}$ shows the mapped fictitious path based on the holographic principle.

only a non-relativistic formula. In Ref. [18], based on the consideration of the loss of the information of the path, it is suggested that

$$
S_{M} \sim \frac{l_{A B}}{\lambda_{M}}
$$

with the reduced Compton wavelength $\lambda_{M}=\hbar / M c$ as an effective resolution of the particle $M$ by spacetime.

There is another way to understand this formula by using the so-called holographic principle. Based on holographic principle [14, 19, 20], all the information inside the sphere are reflected on the spherical surface. In Fig. 2, the mapped fictitious path from $A^{\prime}$ to $B^{\prime}$ shows the information of the path of $M$ inside the sphere. However, when the entanglement entropy is considered by the partial trace of $|\Psi\rangle\langle\Psi|$ with respect to the system $D$, this information of the fictitious path $A^{\prime} \rightarrow B^{\prime}$ is lost in the reduced density matrix, and thus leads to an increase of the entanglement entropy for this displacement from $A$ to $B$.

Of course, we should consider an improvement of Eq. (2) so that it becomes a relativistic expression for curved spacetime and an invariant quantity for any observer. For any 
spacetime path of the particle $M$ inside the sphere, we have the following invariant quantity

$$
\tau_{A B} \sim \int_{A}^{B} d \tau \sqrt{-g_{\mu \nu}\left(X^{\alpha}(\tau)\right) \frac{d X^{\mu}(\tau)}{d \tau} \frac{d X^{\nu}(\tau)}{d \tau}} .
$$

Here $X^{\mu}(\tau)$ is the spacetime coordinate of this particle $M . g_{\mu \nu}$ is the metric tensor of spacetime, while $d \tau$ the proper time. It is tempting to consider the possibility of $S_{M} \sim$ $\tau_{A B}$. On the other hand, we know that the mass $M$ of the particle means a coupling with spacetime. Hence, it is expected that $S_{M} \sim M c^{2}$. This analysis reminds us of the action for particle in general relativity.

To give further connection between the entanglement entropy and the action of $M$, we want to make $M c^{2} \tau_{A B}$ become dimensionless. The dimension of $\int_{A}^{B} d \tau \sqrt{-g_{\mu \nu}\left(X^{\alpha}(\tau)\right) \frac{d X^{\mu}(\tau)}{d \tau} \frac{d X^{\nu}(\tau)}{d \tau}}$ is length, while the dimension of $M c^{2}$ is energy. Hence, we may consider the following dimensionless action for $M$.

$$
S_{M}=\frac{M c^{2}}{E_{p} l_{p}} \int_{A}^{B} d \tau \sqrt{-g_{\mu \nu}\left(X^{\alpha}(\tau)\right) \frac{d X^{\mu}(\tau)}{d \tau} \frac{d X^{\nu}(\tau)}{d \tau}}
$$

Here $E_{p}=\sqrt{\hbar c^{5} /|G|}$ is the Planck energy, while $l_{p}=\sqrt{\hbar|G| / c^{3}}$ is the Planck length. $G$ is the gravitational constant. We use the absolute value of $G$ in $E_{p}$ and $l_{p}$ so that we may give a general consideration of $G$ for both negative and positive values. Because the entanglement entropy is considered based on the fundamental property of spacetime, we use Planck units to make Eq. (6) become dimensionless. In fact, the same consideration can be used to get $S_{0} \sim 4 \pi r^{2} / l_{p}^{2}$ in Eq. (3).

Because the action of the particle $M$ in Einstein's general relativity is [21]

$$
A_{M}=M c^{2} \int_{A}^{B} d \tau \sqrt{-g_{\mu \nu}\left(X^{\alpha}(\tau)\right) \frac{d X^{\mu}(\tau)}{d \tau} \frac{d X^{\nu}(\tau)}{d \tau}}
$$

we have

$$
S_{M}=\frac{A_{M}}{E_{p} l_{p}}
$$

From $E_{p}=\hbar / t_{p}$ and $l_{p}=c t_{p}$ with $t_{p}$ the Planck time, we have the following dimensionless action for matter

$$
S_{M}=\frac{\int_{A}^{B} d \tau \sqrt{-g_{\mu \nu}\left(X^{\alpha}(\tau)\right) \frac{d X^{\mu}(\tau)}{d \tau} \frac{d X^{\nu}(\tau)}{d \tau}}}{\lambda_{M}} .
$$

It is interesting to notice that the same $\lambda_{M}$ appears in the conjecture given by Eq. (4). This gives a strong support to the connection between the entanglement entropy and the action of the particle. 
To consider the physical meaning of this dimensionless action for matter, we consider the case of a rest particle in flat spacetime. In this situation, we have

$$
S_{M}=\frac{\int_{A}^{B} c d t}{\lambda_{M}} .
$$

We see that $\int c d t$ is the length of the path of this rest particle in Minkowski spacetime. Because $\lambda_{M}$ is the Compton wavelength of this particle, it is natural to regard $S_{M}$ as the entropy of this particle. In a sense, the Compton wavelength is the effective resolution of the location of $M$ by spacetime.

We see that $S_{M}$ increases with time, although this particle is at rest in spacetime. There are several reasons for this behavior. (i) When both space and time are considered in a unified way, any particle always has nonzero four velocity. (ii) We consider a thought experiment that the mass of the particle increases very slowly from zero to $M$. The gravitational field of $M$ propagates in spacetime from $M$ with velocity $c$. For infinite universe, this propagation never stops and it is always a dynamic process. This provides another reason for the behavior given by Eq. (10).

We may consider a comparison between $S_{0}$ and $S_{M}$. $S_{0}$ is of the order of $4 \pi r^{2} / l_{p}^{2}$ for a sphere with radius $r$. We consider a proton inside this sphere. If the time $t$ is one second and $r=1 \mathrm{~m}$. We have $S_{M} / S_{0} \approx 2.9 \times 10^{-47}$. Hence, for the usual time scale of the gravitational force we consider, $S_{M}$ is much smaller than $S_{0}$.

We may also consider the non-relativistic approximation in flat spacetime for moving particle. $S_{M}$ in this case becomes

$$
S_{M} \approx \frac{\int c d t}{\lambda_{M}}-\frac{1}{\hbar} \int \frac{1}{2} m \mathbf{v}^{2} d t
$$

Here $\mathbf{v}=d \mathbf{r} / d t$. We see that the second term in this action is the kinetic energy term in the path intergral calculation of probability amplitude for non-relativistic particle. In future work, it would be interesting to consider the entanglement entropy in the path integral formalism.

\section{ENTANGLEMENT ENTROPY OF SPACETIME}

In proceeding section, we consider the entanglement entropy of the particle $M$ inside the sphere. This entanglement entropy originates from the coupling of $M$ with spacetime. It is 
expected that the spacetime will also contribute to an extra entanglement entropy because of the reaction of $M$ on spacetime. Based on the connection of the entanglement entropy $S_{M}$ with the action $A_{M}$, it is natural to consider the situation that the extra spacetime entanglement entropy $S_{\text {spacetime }}$ has similar connection with the so-called Einstein-Hilbert action. The Einstein-Hilbert action for spacetime is

$$
A_{\text {spacetime }} \sim \int d^{4} x \sqrt{-g} R
$$

Here $R$ is the scalar curvature due to the particle. The dimension of $R$ is $1 / L^{2}$. Hence, the dimension of $\int d^{4} x \sqrt{-g} R$ is $L^{2}$.

The dimensionless $S_{\text {spacetime }}$ for the entanglement entropy of spacetime is then

$$
S_{\text {spacetime }}=\frac{\alpha \int d^{4} x \sqrt{-g} R}{l_{p}^{2}} .
$$

Here $\alpha$ is a constant which will be given in due course. From Einstein's field equation

$$
R_{\mu \nu}-\frac{1}{2} g_{\mu \nu} R=\frac{8 \pi G}{c^{4}} T_{\mu \nu}
$$

we have

$$
R=-\frac{8 \pi G}{c^{4}} g_{\mu \nu} T^{\mu \nu}
$$

Here $T^{\mu \nu}$ is the stress-energy tensor of $M$, which is given by

$$
T^{\mu \nu}\left(x^{\alpha}\right)=\frac{M c^{2}}{\sqrt{-g\left(x^{\alpha}\right)}} \int d \tau \frac{X^{\mu}(\tau)}{d \tau} \frac{d X^{\nu}(\tau)}{d \tau} \delta^{(4)}\left(x^{\mu}-X^{\mu}(\tau)\right) .
$$

Here $g=\operatorname{det}\left(g_{\mu \nu}\right)$.

To show the physical meaning of $S_{\text {spacetime, }}$ we give an approximate calculation by approximating $g_{\mu \nu}$ as the case of flat spacetime. Under this approximation, we have

$$
R \approx \frac{8 \pi G M}{c^{2}} \delta^{(3)}\left(\mathbf{x}-\mathbf{r}_{M}\right)
$$

Here $\mathbf{r}_{M}$ is the spatial coordinate of the particle $M$. In this case, we get

$$
S_{\text {spacetime }} \approx 8 \pi \alpha \frac{\int c d t}{\lambda_{M}} .
$$

Compared with Eq. (10), we see that $S_{\text {spacetime }}$ can be also regarded as the entanglement entropy for the spacetime induced by $M$. It is expected that $S_{\text {spacetime }}$ should be the same order of $S_{M}$. This means that $\alpha \sim 1 / 8 \pi$. In the next section, we will show that $\alpha=1 / 16 \pi$. 


\section{ENTANGLEMENT ENTROPY AND ACTION PRINCIPLE}

We consider two particles $M$ and $m$ in spacetime. Because the overall entanglement entropy $S$ should be the sum of $S_{\text {spacetime }}, S_{M}$ and $S_{m}$, we have

$$
S=S_{\text {spacetime }}+S_{M}+S_{m}
$$

Hence, we have

$$
S=\frac{\alpha}{l_{p}^{2}} \int d^{4} x \sqrt{-g} R+\frac{M c^{2}}{E_{p} l_{p}} \int d \tau \sqrt{-g_{\mu \nu} \frac{d X_{1}^{\mu}}{d \tau} \frac{d X_{1}^{\nu}}{d \tau}}+\frac{m c^{2}}{E_{p} l_{p}} \int d \tau \sqrt{-g_{\mu \nu} \frac{d X_{2}^{\mu}}{d \tau} \frac{d X_{2}^{\nu}}{d \tau}} .
$$

Here $X_{1}^{\mu}$ is the spacetime coordinate for particle $M$, while $X_{2}^{\mu}$ is the spacetime coordinate for $m$. It can be rewritten as

$$
S=\frac{1}{E_{p} l_{p}}\left[\frac{\alpha c^{4}}{|G|} \int d^{4} x \sqrt{-g} R+M c^{2} \int d \tau \sqrt{-g_{\mu \nu} \frac{d X_{1}^{\mu}}{d \tau} \frac{d X_{1}^{\nu}}{d \tau}}+m c^{2} \int d \tau \sqrt{-g_{\mu \nu} \frac{d X_{2}^{\mu}}{d \tau} \frac{d X_{2}^{\nu}}{d \tau}}\right]
$$

By setting $\alpha=1 / 16 \pi$, the expression in the square brackets is the action in general relativity.

We know that the system evolution tends to maximize the entropy, and this leads to the action principle in general relativity, which will then give us Einstein's field equation. Here we show the microscopic mechanism of the action principle in general relativity and the sum rule for various contributions to the action.

It is worthwhile to point out that the application of the entanglement entropy gives us the chance to say something more about the fundamental property of gravitational force. In Einstein's general relativity, the attractive characteristic is imposed on by the observation, rather than by rational consideration. Now, we have the chance to consider the attractive characteristic of gravitational force for classical particles. The sum rule of entanglement entropies lead to Eq. (21). The positive value of entanglement entropy means that $M>0$, $m>0$ and the absolute value of $G$ in Eq. (21). Of course, it is not necessary to retain this symbol of absolute value by setting $G>0$. In this case, from the action principle to get the Einstein's field equation, the gravitational force between two classical particles $M$ and $m$ must be attractive.

\section{SUMMARY AND DISCUSSION}

In summary, we give a general approach to calculate the entanglement entropy for spacetime and matter based on the action in general relativity. This entanglement entropy pro- 
vides a mechanism for the action principle and attractive characteristic of gravitational force for classical particles. In future work, it would be interesting to consider the relevant entanglement entropy for dark energy [22, 23], dark matter and black hole [24, 25].

\section{Acknowledgments}

This work was supported by NSFC 11175246, 11334001.

[1] C Holzhey, F Larsen, and F Wilczek. Geometric and renormalized entropy in conformal field theory. Nucl. Phys. B, 424(3):443-467, 1994.

[2] K Skenderis and M Taylor. The fuzzball proposal for black holes. Phys. Rep., 467(4-5):117-171, 2008.

[3] D D Blanco, H Casini, L Y Hung, and R C Myers. Relative entropy and holography. J. High Energy Phys., (8):060, 2013.

[4] S W Hawking, G T Horowitz, and S F Ross. Entropy, area, and black-hole pairs. Phys. Rev. $D, 51(8): 4302-4314,1995$.

[5] A Almheiri, D Marolf, J Polchinski, and J Sully. Black holes: complementarity or firewalls? J. High Energy Phys., (2):062, 2013.

[6] M Van Raamsdonk. Building up spacetime with quantum entanglement. Gen. Rel. Gravit., 42(10):2323-2329, 2010.

[7] N Lashkari, M B McDermott, and M Van Raamsdonk. Gravitational dynamics from entanglement thermodynamics. J. High Energy Phys., (4):195, 2014.

[8] G Resconi, I Licata, and D Fiscaletti. Unification of quantum and gravity by nonclassical information entropy space. Entropy, 15(9):3602-3619, 2013.

[9] Greg Ver Steeg and Nicolas C. Menicucci. Entangling power of an expanding universe. Phys. Rev. D, 79(4):044027, 2009.

[10] P. A. Zizzi. Holography, quantum geometry, and quantum information theory. Entropy, 2:3969, 2000.

[11] Paola Zizzi. Entangled spacetime. Mod. Phys. Letts. A, 33(29), 2018. 
[12] Claudio Cremaschini and Massimo Tessarotto. Quantum-Wave Equation and Heisenberg Inequalities of Covariant Quantum Gravity. Entropy, 19(7):339, 2017.

[13] Michael A. Nielsen and Isaac L. Chuang. Quantum computation and quantum information. Cambridge University Press, Cambridge, 2011.

[14] R Bousso. The holographic principle. Rev. Mod. Phys., 74(3):825-874, 2002.

[15] M Srednicki. Entropy and area. Phys. Rev. Lett., 71(5):666-669, 1993.

[16] J Eisert, M Cramer, and M B Plenio. Colloquium: area laws for the entanglement entropy. Rev. Mod. Phys., 82(1):277-306, 2010.

[17] E Verlinde. On the origin of gravity and the laws of Newton. J. High Energy Phys., (4):029, 2011.

[18] H W Xiong. Repulsive gravitational effect of a quantum wave packet and experimental scheme with superfluid helium. Front. Phys., 10(4):1-9, 2015.

[19] C R Stephens, G 't Hooft, and B F Whiting. Black-hole evaporation without information loss. Class. Quantum Grav., 11(3):621-647, 1994.

[20] L Susskind. The world as a hologram. J. Math. Phys., 36(11):6377-6396, 1995.

[21] S Weinberg. Cosmology. Oxford, New York, 2008.

[22] P J E Peebles and B Ratra. The cosmological constant and dark energy. Rev. Mod. Phys., $75(2): 559-606,2003$.

[23] H W Xiong. On the quantitative calculation of the cosmological constant of the quantum vacuum. arXiv:1805.10440, May 2018.

[24] S W Hawking. Particle creation by black-holes. Commun. Math. Phys., 43(3):199-220, 1975.

[25] J D Bekenstein. Black holes and entropy. Phys. Rev. D, 7(8):2333-2346, 1973. 